UCRL-JC-114214

PREPRINT

\title{
Design Study of a Microwave Driver for a Relativistic
Klystron Two-Beam Accelerator 0
}

T. L. Houck

This paper was prepared for submittal to 1993 Particle Accelerator Conference May 17-20, 1993

Omni Shoreham Hotel, Washington, DC

May 1993

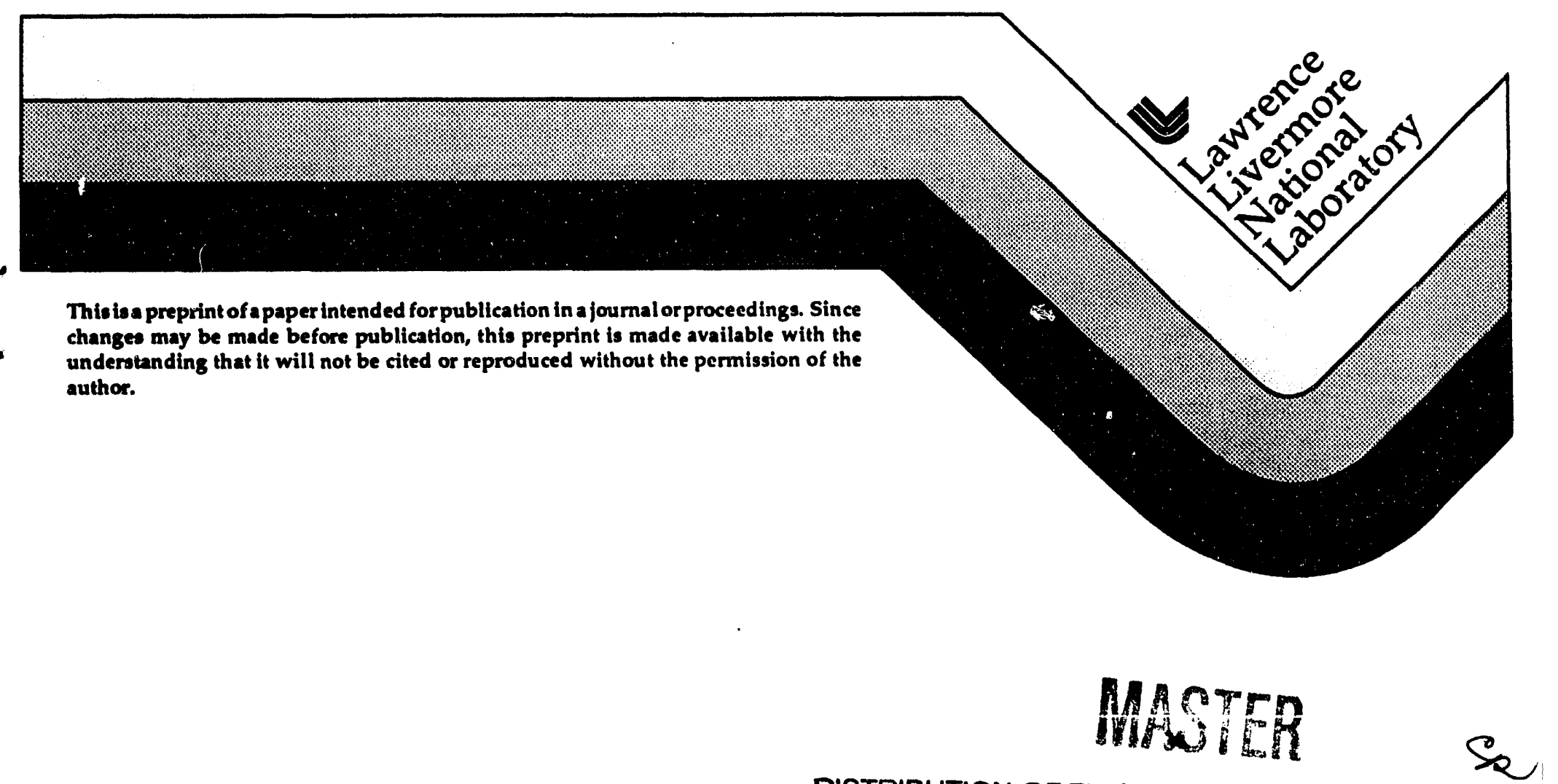




\section{DISCLAIME:}

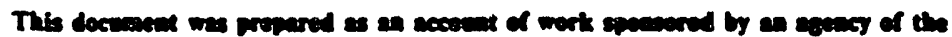

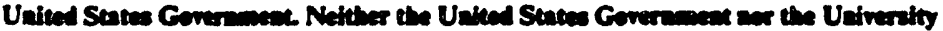

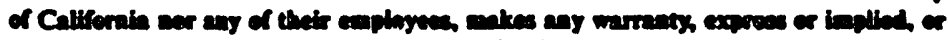

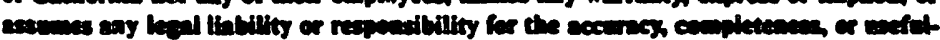

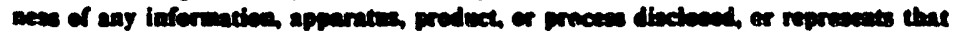

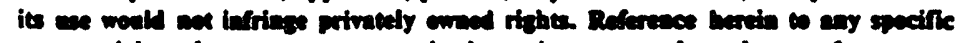

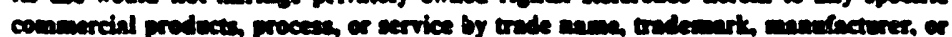

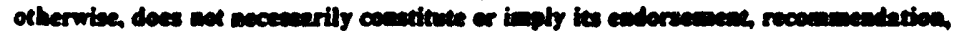

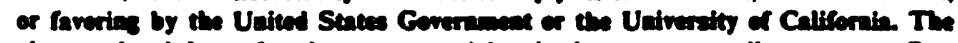

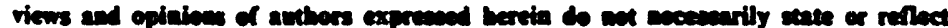

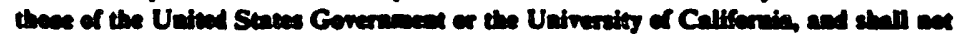

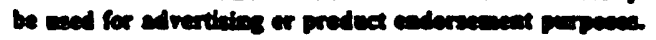




\title{
Design Study of a Microwave Driver for a Relativistic Klystron Two-Beam Accelerator*
}

\author{
T.L. Houck \\ Lawrence Livermore National Laboratory \\ University of California, Livermore, California 94550 USA
}

\begin{abstract}
In two-beam accelerators, the reacceleration of a modulated drive beam can enable high conversion efficiency of electron beam energy to $\mathrm{rf}$ energy. However, the stability issues involved with the transport of high current electron beams through $\mathrm{rf}$ extraction structures and induction accelerator cells are critical. We report on theoretical studies and computer simulations of a two-beam accelerator design using traveling-wave extraction structures. Specific issues addressed include regenerative and cumulative transverse instabilities.
\end{abstract}

\section{Introduction}

A collaboration between the Lawrence Livermore National Laboratory's (LLNL) Microwave Source Facility and the Lawrence Berkeley Laboratory's Collider Physics Group has been studying the feasibility of a Relativistic Klystron-Two Beam Accelerator (RK-TBA) as a possible linear collider. In a RK-TBA, one beam line is a high-gradient rf linac which accelerates electrons or positrons to very high energies. The second beam line, the subject of this paper, is an induction linac which includes microwave generating structures located at regular intervals along the beam line. These structures extract energy in the form of microwaves which are then used to drive the rf linac. In a RK-TBA design, the microwave generating structures are rf structures, e.g. standing-wave cavities or traveling-wave structures (TWS). Experiments at LLNL have shown that traveling-wave structures are capable of producing the desired high-power microwave pulses (100's MW per output). ${ }^{1}$ However, these experiments also indicated that the major difficulty in designing a feasible RK-TBA is the transverse instabilities resulting from the propagation of kiloamperes of average current through the narrow apertures of $x$-band microwave structures. ${ }^{2}$ This paper addresses both analytical and numerical modeling performed to study damping of the transverse instability in such systems.

\section{Analytical Modeling}

To model the RK-TBA, I have used Caporaso's technique ${ }^{3}$ for studying the growth of transverse insta-

*The work was performed under the auspices of the U.S. Department of Energy by Lawrence Livermore National Laboratory under contract W-7405-ENG-48. bilities in induction accelerators. The microwave extraction cavities are treated as a continuous distribution along the drive beam of the RK-TBA. The transverse instability is assumed to be due to the excitation of a single dipole resonant mode with frequency $\omega_{0}$. Within this frame work the asymptotic behavior of the transverse instability can be studied using the well-known multicavity model equations: 4,5

$$
\begin{gathered}
\left(\frac{\partial^{2}}{\partial t^{2}}+\frac{\omega_{0}}{Q} \frac{\partial}{\partial t}+\omega_{0}^{2}\right) \Delta=\omega_{0}^{2} G \xi / \gamma \\
\frac{\partial}{\partial z}\left[\gamma \frac{\partial \xi}{\partial z}\right]+\gamma k_{p}^{2} \xi=\gamma \Delta,
\end{gathered}
$$

where $\xi$ is the transverse displacement of the beam centroid from the axis. $\Delta \equiv \Delta \mathrm{p}_{\perp} /\left(\mathrm{L}_{\mathrm{g}} \mathrm{p}_{\mathrm{z}}\right)$ is the normalized $z$-averaged transverse angular change of the beam centroid per unit length, $L_{g}$ is the cavity spacing, $\Delta p_{\perp}$ is the change in transverse momentum, and $p_{2}$ is the longitudinal momentum. The strength of the coupling between the beam and the dipole field is represented by the term $G \equiv c \omega_{0}\left(Z_{\perp} / Q\right) I /\left(L_{g} I_{o}\right)$, where $I_{o}=\mathrm{mc}^{3} / \mathrm{e}=$ $17 \mathrm{kA}$. For solenoidal focusing $\mathrm{k}_{\beta}$ should be interpreted as one half of the cyclotron wave number and $\xi$ is equal to the phasor $x+i y$. In the RK-TBA concept the average energy of the induction beam remains constant over an extraction-reacceleration cycle. In the continuous distribution approximation I will treat $\gamma$ as a constant.

Equations (1) and (2) can be solved by Fourier transforming from $t$ to $\omega$ and using the WKB method. The transform inversion to recover $\xi$ can only be done analytically in a few special cases of initial/boundary conditions. However, general information on the growth of the transverse instability can be obtained if two simplifying assumptions are made;

$$
k_{\beta}^{2}>>\frac{\omega_{0}^{2} G}{\gamma\left(\omega_{0}^{2}-\omega^{2}-i \omega_{0} \omega / Q\right)^{\prime}}
$$

and the instability growth is sharply peaked near the resonance, $\omega=\omega_{0}$. The solution can then be approximated as:

$$
\xi \approx \frac{A\left(\omega_{0}\right) \omega_{0}}{2 \pi Q} \exp \left(-i \omega_{0} t+i \int_{0}^{z} k_{\beta}-\frac{i G Q}{2 \gamma k_{\beta}} d z\right)-\exp \left[\frac{G Q z}{2 \gamma k_{\beta}}\right],
$$

where $A\left(\omega_{0}\right)$ is determine by boundary conditions. Equation (3) exhibits the exponential growth with distance that is characteristic of this instability.

Phase mixed damping due to a spread in energy across the beam can be included in the analysis by using 
the techniques of hose instability theory. ${ }^{6}$ The beam is divided into "beamlets" with different energies, oscillation amplitudes, and betatron wavelengths such that $k_{\eta}^{2}=\eta k_{\beta}^{2}$, where $k_{\beta}$ is the maximum wave number, $0 \leq \eta \leq 1$, and $\xi_{\eta}$ are the associated oscillation amplitudes. The model equations are

$$
\begin{aligned}
& \left(\frac{\partial^{2}}{\partial t^{2}}+\frac{\omega_{0}}{Q} \frac{\partial}{\partial t}+\omega_{0}^{2}\right) \Delta=\omega_{0}^{2} G(\xi) / \gamma \text {, and } \\
& \frac{\partial^{2} \xi_{\eta}}{\partial z^{2}}+k_{\eta}^{2} \xi_{\eta}=\Delta, \text { where } \\
& \langle\xi\rangle=\int_{0}^{1} g(\eta) \xi_{\eta} d \eta, \gamma=\int_{0}^{1} g(\eta) \gamma_{\eta} d \eta \text {, and } \int_{0}^{1} g(\eta) d \eta=1 \text {. }
\end{aligned}
$$

For this analysis, let the distribution function be:

$$
g(\eta)=\left\{\begin{array}{c}
0 \text { for } 0 \leq \eta<1-\varepsilon \\
1 / \varepsilon \text { for } 1-\varepsilon<\eta \leq 1
\end{array} .\right.
$$

A dispersion relationship can be obtained from equations (4) and (5) by Fourier transforming $\omega$ to $t$ and $z$ to $k$ and using the distribution in equation (6):

$$
1=\frac{\omega_{0}^{2} G}{\gamma\left(\omega_{o}^{2}-\omega^{2}-i \omega_{0} \omega / Q\right) \varepsilon k_{\beta}^{2}} \ln \left[\frac{k_{\beta}^{2}-k^{2}}{k_{\beta}^{2}(1-\varepsilon)-k^{2}}\right] \text {. }
$$

I will again assume that the greatest growth in the instability occurs when $\omega=\omega_{0}$, and then solve for $k$ :

$$
k^{2}=k_{\beta}^{2}\left(1+\frac{\varepsilon e^{i \phi}}{1-e^{i \phi}}\right)=k_{\beta}^{2}\left(1-\frac{\varepsilon}{2}+i \frac{\varepsilon}{2} \cot \left(\frac{\phi}{2}\right)\right)
$$

where $\phi=\varepsilon \gamma \mathrm{k}_{\mathrm{s}}^{2} /(\mathrm{GQ})$. If $\phi=\pi$, $\mathrm{k}$ will be a purely real number and $\xi$ will not exhibit exponential growth.

\section{Numerical Modeling}

The Beam Breakup (BBU) Code $^{7}$ developed at LLNL was used to numerically study the transverse instability in a microwave generator comprised of many equally spaced traveling-wave structures (TWS). The BBU Code assumes a single dipole cavity mode is dominant and the $x$-polarization of the electric field in the $\mathrm{n}^{\text {th }}$ cavity can be expressed as

$$
\vec{E}_{n}(\vec{r}, t)=f_{n}(t) \vec{\xi}_{n}(\vec{r}) e^{i \omega t},
$$

where $\vec{\xi}_{n}$ denotes an eigenmode with eigenfrequency $\omega_{n}$. Here $\omega$ denotes a characteristic frequency of the generator assumed near the transverse instability resonance. It is possible to show that the excitation amplitudes $f_{n}$ are governed by the following circuit equations:

$$
\begin{aligned}
& \frac{\partial^{2} f_{n}}{\partial t^{2}}+\left(\frac{\omega_{n}}{Q_{n}}-2 i \omega\right) \frac{\partial f_{n}}{\partial t}+\left(\omega_{n}^{2}-\omega^{2}-\frac{i \omega \omega_{n}}{Q_{n}}\right) f_{n}= \\
& K_{n}^{n-1} f_{n-1}+K_{n}^{n+1} f_{n+1}+\frac{\omega_{n}^{3}}{\varepsilon c^{2}}\left(\frac{Z_{\perp}}{Q}\right)_{n} \frac{\partial I x}{\partial t} e^{-i\left(\omega t+\phi_{n}\right)},
\end{aligned}
$$

where $Q_{n}$ is the quality factor of the $n^{\text {th }}$ cavity, $K_{n}^{n \pm 1}$ denotes the coupling of the $n$ and $n+1$ cavities, $I$ is the current, $x$ is the transverse displacement of the beam centroid in the $x$ direction from the center line, $\phi$ is a phase advance, and $Z_{\perp}$ is the transverse impedance. $A$ second equation is used for the $y$-polarization as well as single particle equations of motion in the $x$ and $y$ directions. While equation (10) is similar to equation (4), there are several features in the BBU Code necessary for modeling a realistic structure:

a. RF structures are treated as separate entities with specific rf properties,

b. RF structures have a finite longitudinal length,

c. Electromagnetic coupling can exist between adjacent rf cavities (needed for TWS cells),

d. Variable current profiles can be used, and

e. Actual solenoidal fields included for focusing. The electromagnetic coupling of cells allows the effect of regenerative $\mathrm{BBU}$ to be modeled. A limitation is that the beam is treated as a series of rigid disks. Thus, beam parameters such as current and energy can only be varied longitudinally.

Modeling parameters used in the simulations presented below are given in Table 1 . For some simulations five different configurations of TWS's were used. Two configurations were modeled after TWS's tested during the Choppertron experiments, ${ }^{1}$ and a third after a structure to be tested in the near future. The final two configurations have rf characteristics bracketed by the others. De-Q-ing circuits ${ }^{8}$ mentioned below refer to a technique for damping the dipole modes in TWS's used at LLNL. TWS's that are de-Q-ed are simulated by reducing the $Q$ of the first two cells to about 20 . In all the simulations the current is modulated at $11.4 \mathrm{GHz}$, the $2 \pi / 3$ longitudinal mode of the TWS's, with each microbunch covering $120^{\circ}$ of phase. Beam energy is kept constant, although it is possible to simulate energy extraction and reacceleration with the BBU Code.

TABLE 1.

TWS Modeling Parameters in Simulations

\begin{tabular}{lc|}
\hline dipole mode & lower "HEM 11 branch" \\
frequency $(\mathrm{GHz})$ & $13.75,13.675,13.6,13.525,13.45$ \\
phase advance & $142.5^{\circ}$ per cell \\
\# cells per TWS & 7 \\
IWS electrical length & $6.1278 \mathrm{~cm}$ \\
cell aperture (mm) & $13,13.5,14,15,16$ \\
group velocity & $0.12 \mathrm{c}, 0.13 \mathrm{c}, 0.14 \mathrm{c}, 0.15 \mathrm{c}, 0.16 \mathrm{c}$ \\
Qwall (cells) & $3000(2), 7000(5)$ \\
Qext (damped cells) & 20 \\
$Z_{1} / Q_{\text {wall }}$ & $18.33 \Omega /$ cell \\
TWS spacing & $50 \mathrm{~cm}(\mathrm{center}$ to center) \\
dc/rf currents & $650 / 600 \mathrm{amps}$ \\
pulse length & $110 \mathrm{~ns}$ (includes $5 \mathrm{~ns} \mathrm{ramp} \mathrm{time)}$ \\
beam energy & $10 \mathrm{MeV}$ \\
initial offset & $0.1 \mathrm{~mm}$ \\
solenoidal field $\left(\mathrm{B}_{\mathbf{z}}\right)$ & $8 \mathrm{KG}$ \\
\hline
\end{tabular}

Figure 1 illustrates the effect of adding de-Q-ing circuits to the first two cells of the TWS's. The displacement of the beam centroid is measured midway between TWS's. Points are plotted whenever the centroid displacement has doubled after passing 
through a TWS. The abrupt growth in the case of no de-Q-ing indicates the current was above the threshold for regenerative BBU in an individual TWS. With sufficient damping, as in the de-Q-ed case, cumulative BBU is the principle mechanism for instability growth. Figure 2 shows the effect of varying structures along the beam line (staggered tuning). For example the curve "5 TWS" represents a beam line comprised of repeating sets of five different TWS's. Structures are de-Q-ed in all cases. This effect is similar to lowering the $Q$ for a single configuration. Figure 3 shows the effect of phase

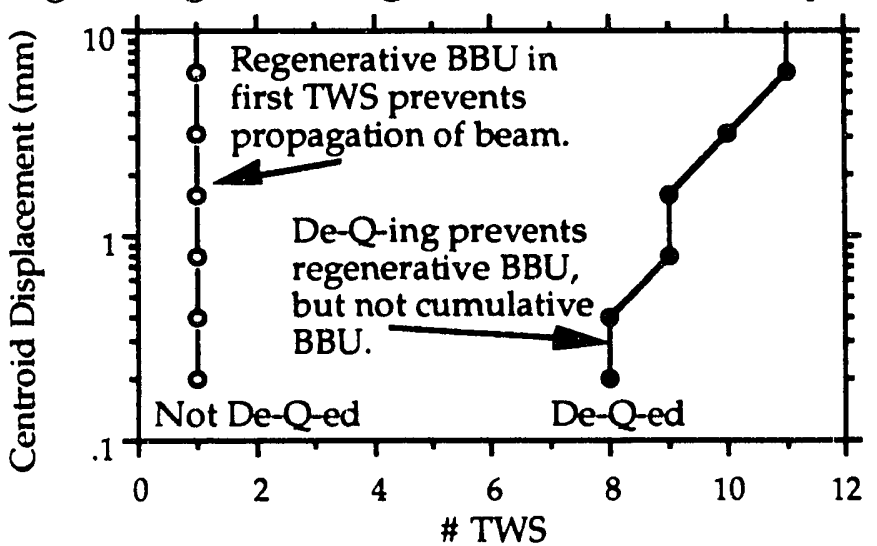

Figure 1. Effect of the de-Q-ing circuit.

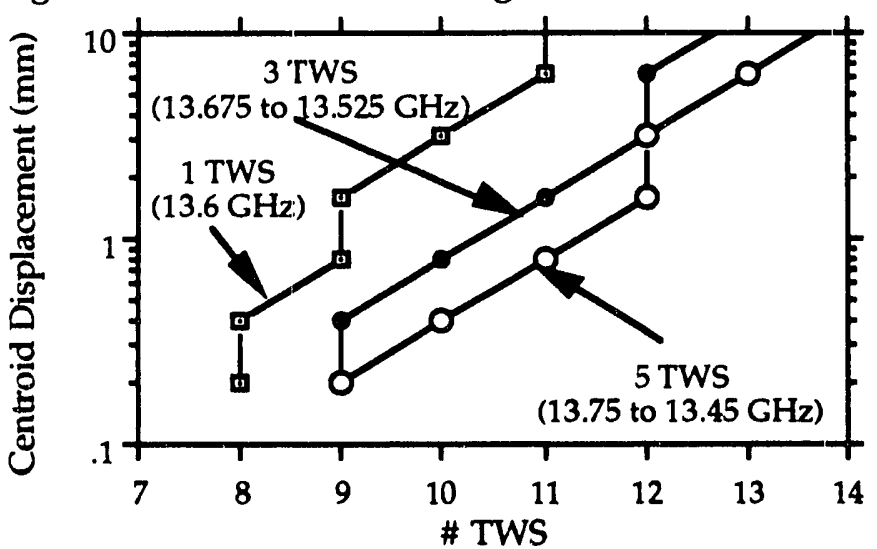

Figure 2. Effect of staggered tuning.

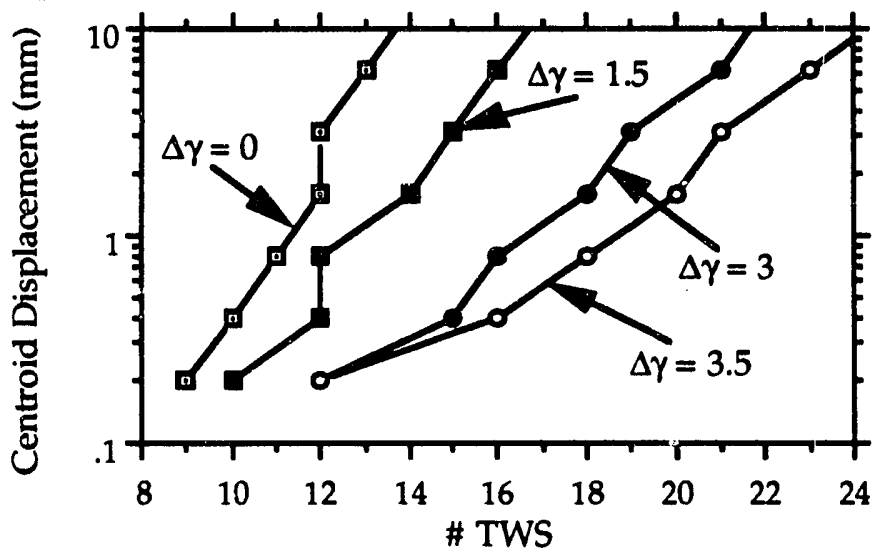

Figure 3. Effect of phase mixing due to energy spread. mixed damping due to a longitudinal variation in energy along the beam (modulated at $11.4 \mathrm{GHz}$ ). Five different de-Q-ed TWS's are used in all cases. Larger spreads in gamma, $\Delta \gamma$, would require greater resolution (thinner current disks) for accuracy.

\section{Conclusions}

It is essential to sufficiently damp the BBU resonances within individual TWS's to prevent the onset of regenerative BBU. It is also necessary to limit electromagnetic coupling between adjacent TWS's to avoid regenerative BBU between TWS's. This condition was assumed in the simulations. Phase mixing is the best means of defeating the cumulative instability, although large energy spreads will make beam transport more difficult. To further increase the practical number of rf output structures in an RK-TBA, I will examine more aggressive de-Q-ing, different TWS geometry's, and the use of standing-wave cavities. Also, additional work needs to be accomplished to determine a more realistic current density distribution and to model two-dimensional phase mixing.

\section{Acknowledgments}

I would like to thank G. Caporaso, J. Haimson, V.K. Neil, D. Whittum, and S. Yu for their advice and insights on beam instabilities. I thank G. Westenskow for guidance and encouragement.

\section{References}

[1] T.L. Houck and G.A. Westenskow, "Status of the Choppertron Experiments," Proc. 16 ${ }^{\text {th }}$ Int'l LINAC Conf., Aug , 1992, pp. 498-500.

[2] G.A. Westenskow, et. al., "Transverse Instabilities in a Relativistic Klystron Two-Beam Accelerator," Proc. $16^{\text {th }}$ Int'l LINAC Conf., Aug, 1992, pp.263-267.

[3] G.J. Caporaso, "The Control of Beam Dynamics in High Energy Induction LINACS," Proc. $13^{\text {th }}$ Int'l LINAC Conf., June, 1986, pp. 17-21.

[4] W.K.H. Panofsky and M. Bander, "Asymptotic Theory of Beam Break-Up in Linear Accelerators," Rev. Sci. Instrum. 39, 206 (1968).

[5] V.K. Neil and R.K. Cooper, "Coherent Instabilities in High Current Linear Induction Accelerators," Part. Accel. 1, 111 (1970).

[6] E.P. Lee, "Resistive Hose Instability of a Beam with the Bennett Profile," Physics Fluids 21, Aug 1978, pp. 1327-1343.

[7] T.L. Houck, et. al., "BBU Code Development for High-Power Microwave Gencrators," Proc. $16^{\text {th }}$ Int'l LINAC Conference, Aug, 1992, pp. 495-497.

[8] J.Haimson and B.Mecklenburg, "Suppression of beam induced pulse shortening ...," SPIE Vol. 1629 Intense Microwave and Part. Beams III (1992), pp. 209-219. 


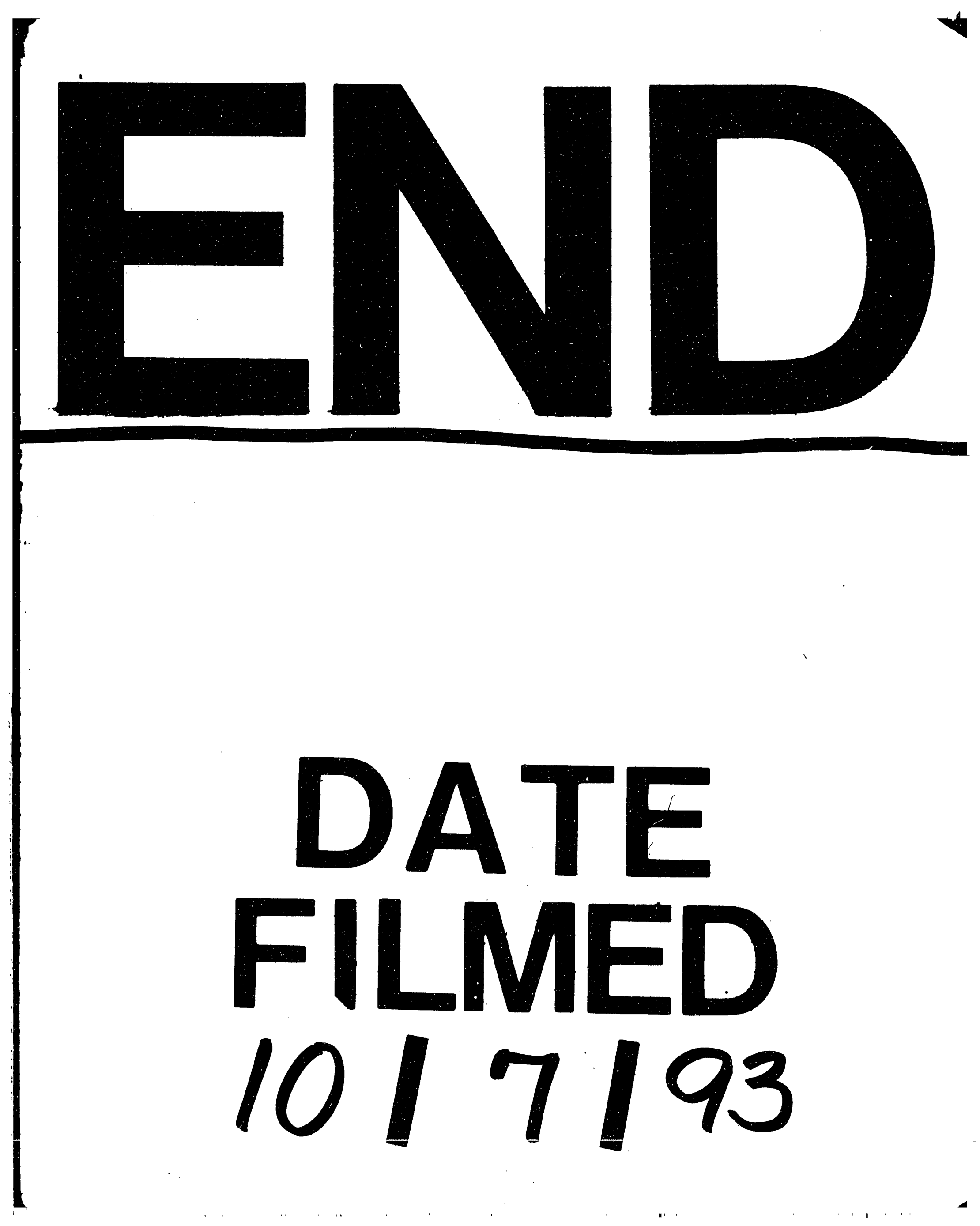


